\title{
Teaching Mechanics Concepts Using a Motion Analysis System
}

\section{Dr. Brian P. Self, California Polytechnic State University, San Luis Obispo}

Brian Self obtained his B.S. and M.S. degrees in Engineering Mechanics from Virginia Tech, and his Ph.D. in Bioengineering from the University of Utah. He worked in the Air Force Research Laboratories before teaching at the U.S. Air Force Academy for seven years. Brian has taught in the Mechanical Engineering Department at Cal Poly, San Luis Obispo since 2006. During the 2011-2012 academic year he participated in a professor exchange, teaching at the Munich University of Applied Sciences. His engineering education interests include collaborating on the Dynamics Concept Inventory, developing model-eliciting activities in mechanical engineering courses, inquiry-based learning in mechanics, and design projects to help promote adapted physical activities. Other professional interests include aviation physiology and biomechanics.

\section{Sonya Dick, California State Polytechnic University, San Luis Obispo}

Sonya Dick is a Senior Mechanical Engineering Student at California Polytechnic State University SLO. This is her second year working at the Human Motion Biomechanics Lab. As a research assistant, she helps create and teach interdisciplinary laboratories for undergraduate kinesiology and engineering students. Her work also involves creating simulations of a wide range of devices for the use of educational modules.

\section{Mr. Jay Tyler Davis II, California Polytechnic State University, San Luis Obispo}

Undergraduate Mechanical Engineering student at Cal Poly San Luis Obispo.

\section{Mr. Bradley Wash, California Polytechnic University, San Luis Obispo}

Human Motion Biomechanics Lab 


\title{
Teaching Mechanics Concepts Using a Motion Analysis System
}

\begin{abstract}
Homework-style dynamics problems are typically analyzed "at an instant in time", or possibly in two specific positions. This "snapshot dynamics" approach may hinder students' ability to consider the time-varying nature of dynamic systems. Some teaching strategies to overcome this include computer simulations and the use of videos, but only a few researchers have utilized motion analysis systems or other instrumentation in their dynamics courses.

One of the goals of our recent Keck Foundation grant is to incorporate our motion analysis system into courses in engineering and kinesiology. To date, we have utilized three different assignment versions in our dynamics course: (a) allowing students to select their own artefact for analysis, (b) supplying six specific artefacts for analysis, and (c) interfacing with a Kinesiology class on motor learning to provide "expert" engineering advice on performance measures of novice jugglers versus advanced jugglers.

We have also incorporated the lab into a Kinesiology biomechanics course, as well as two biomechanics courses within the College of Engineering (one in the Mechanical Engineering Department and one in the Biomedical Engineering Department). Finally, a graduate level architectural engineering course used the lab to analyze two and three degree-of-freedom building models to examine responses to different base inputs (including simulated real earthquake data).
\end{abstract}

Students were asked to fill out surveys to determine their subjective experiences of using the lab. Response was generally positive, with biomechanics courses tending to rate the experience more favorably than other students.

\section{Introduction}

By their very nature, textbook problems and examples are static. Although graphs and stop motion photography can be used to infer continuous motion, it can still be difficult to get students to appreciate the changing velocities and accelerations involved in the motion of particles and rigid bodies. To help overcome this, we have incorporated the use of an advanced motion analysis system into several of our courses at Cal Poly.

The use of motion analysis is nothing new - in fact the physics community has been using such technology for years [1-3]. Some investigators have worked to develop their own motion analysis software [4] to get around the high costs of most motion capture systems, and others have recognized the advanced image processing capabilities of MatLab to perform motion tracking [5] (http://biomech.web.unc.edu/dltdv/). New apps and free software such as Tracker (http://physlets.org/tracker/) have allowed even more widespread use of video analysis in the classroom. 
We have some slightly different objectives than most of the researchers mentioned above. Although we perform a lot of disciplinary research in the Human Motion Biomechanics Laboratory (HMBL), the majority of the use is during the summer. During the school year, there are many down times when the lab could be used as a teaching tool in a variety of courses. As part of a Keck Foundation grant, we wanted to (a) increase the multidisciplinary nature of the laboratory by advertising its capabilities to a wide range of students, (b) expose students to advanced experimental techniques that they could use in the future, (c) recruit future undergraduate and graduate students to become research assistants, and (d) find ideas that could be used for future online learning modules for courses like dynamics and biomechanics.

\section{Laboratory Setup}

The HMBL, shown in Figure 1, includes a motion capture system that has twelve near infrared cameras that track the location of retroreflective markers using the video processing software, Cortex (Motion Analysis Corporation, Santa Rosa, CA). The tracking provides position data in three dimensions, and the software can calculate velocities and accelerations, as well as angular position, velocity, and acceleration. For most of the assignments, we have chosen to only provide students with position data so that we can teach them some basic numerical differentiation techniques. The HMBL also has four force plates for kinetic data collection of ground reaction forces.

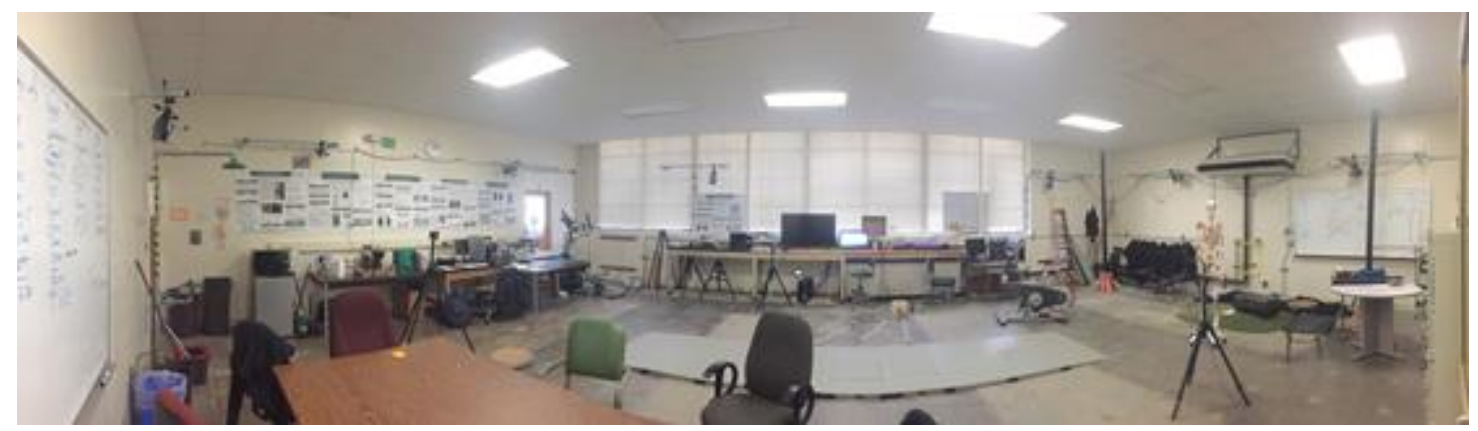

Figure 1. Panoramic image of the Cal Poly Human Motion Biomechanics Laboratory.

\section{Course Project Descriptions}

\section{Dynamics}

Currently we have developed two primary versions of a "lab" for engineering dynamics. In the first implementation, we allowed students to choose their own physical systems to analyze. Participant-directed learning is thought to increase student motivation by allowing them to form linkages between their projects and their previous experiences [6]. For this assignment, students were asked to individually video an "everyday life" example of particle motion and to describe the motion and/or kinetic principles that could be applied to analyze it. They were told that it had to be portable and to make sure it could fit into our lab. Then, in instructor assigned teams of 3-4, students chose a single system to analyze. The assignment was framed in terms of a Model Eliciting Activity [7], where students were asked to develop a multi-media example problem for 
a fictitious book publisher. The students analyzed objects such as a fishing pole, a turntable, a downhill ski, dropping sports balls, and a skateboard.

To provide more structure for the students, and to reduce the workload for our laboratory assistants, our second iteration involved six pre-selected experiments. This iteration was later in the quarter, so most of the physical artefacts involved rigid bodies. The experiments included a bicycle, an elliptical exercise machine, someone walking, someone on a rowing machine, a Trebuchet catapult, and a Geneva mechanism. Students signed up for a specific timeslot, all of which were assigned to one of the six experiments. We don't really know how the students made their choices - if it was based on the times when they were available, the specific experiment, or some other reason (e.g., wanting to sign up with a friend). Pictures of several of the experiments are shown in Figure 2.

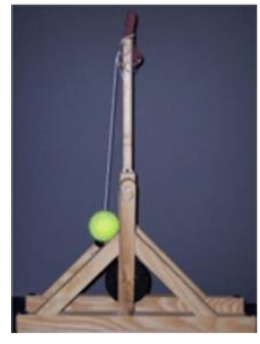

(a)

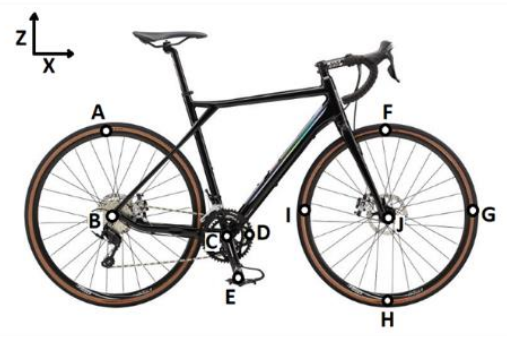

(b)

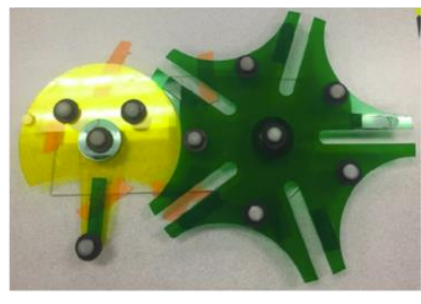

(c)

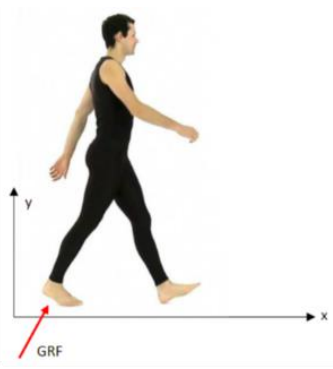

(d)

Figure 2. Dynamics assignment. (a) Trebuchet, (b) bicycle, (c) Geneva Mechanism, (d) gait.

As reported previously, we have used the elliptical machine, the rowing machine, and the gait analysis in an intermediate dynamics course, where students used Matlab to perform advanced analysis and animation of the different motions [8].

\section{Kinesiology: Motor Learning and Control}

An advanced course in the Kinesiology Department at Cal Poly typically uses juggling to demonstrate the acquisition of motor skills through training. In the past, they would have both novice and expert student jugglers conduct their experiment, practice for four weeks, and then repeat their "experimental trials." The only dependent variable was how many balls they could catch and release in a row. During a discussion of potential research collaborations, we decided to bring the kinesiology students into the HMBL and record their motions and that of the balls during the juggling trials. We provided the kinesiology students with graphs of the positions of the balls, shoulder, elbow, and wrist in the $\mathrm{x}, \mathrm{y}$, and $\mathrm{z}$ coordinates as a function of time. In Figure $3 a$, the $x-y-x$ location of the elbow is shown below a freeze frame of an animation. An example output of the height of the three balls is shown in Figure 3b. As reported at a recent conference, during one quarter we paired dynamic students with kinesiology students to try to develop an algorithm that would help determine the juggling level for different participants [9]. 


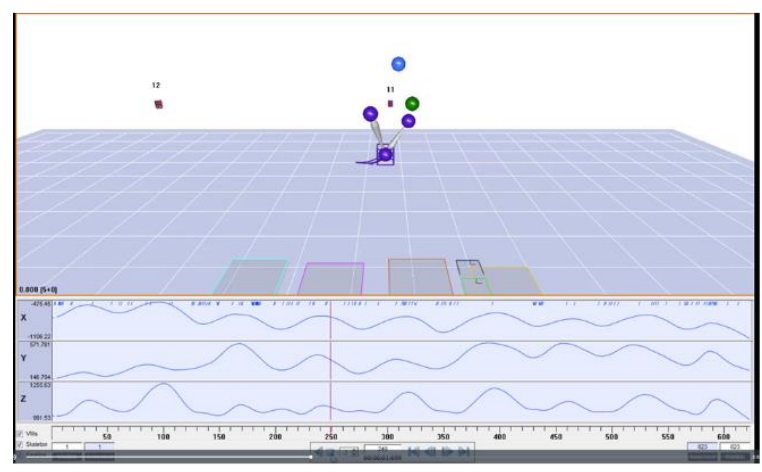

(a)

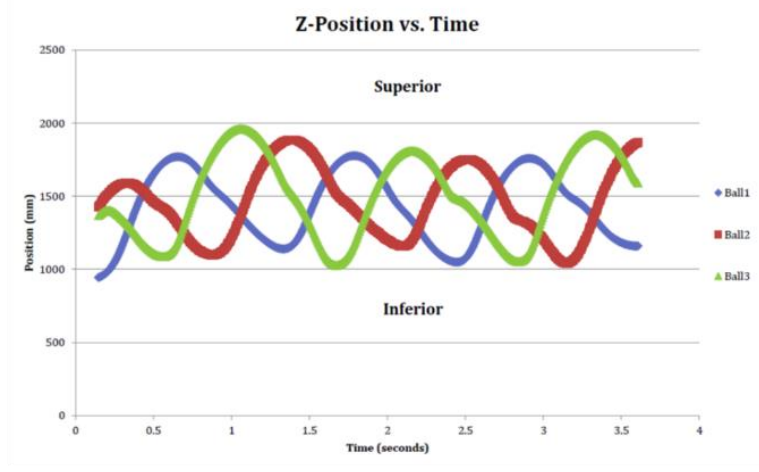

(b)

Figure 3. Cortex animation with elbow position vs time. (b) xyz location of three different balls.

Kinesiology: Biomechanics

The Kinesiology Department has a very basic motion analysis laboratory that uses much older video-based technology and can only analyze two dimensional motion. They only analyzed gait in their kinematics lab assignment, and students did not get exposure to the most current technology. During the tour of the HMBL, the kinesiology students were introduced to the different research projects being conducted in the lab, which include analysis of youth baseball pitchers; determining knee loads in below-tibia amputees during cycling, elliptical exercise, and rowing; and examining back loads during strawberry picking. During the actual laboratory assignment, students first practiced proper placement of the kinematic markers on anatomical landmarks. Then, different students walked across the forceplate using normal gait and then using a knee brace. A second set of experiments had them analyze cycling motion when the seat was at a proper height, when it was too low, and when it was too high. The three dimensional analysis, plus the animation, allowed the students to visualize complex motions that they could not view in their two-dimensional lab.

\section{Biomedical Engineering: Biomechanics}

The Biomedical Engineering Department offers a biomechanics course that is fairly broad in scope. It includes the mechanics of biofluids, application of continuum mechanics to biological tissues, as well as traditional rigid body kinetics. The development of our HMBL prompted the instructor to add a "jumping" lab, where students also use the program OpenSim to investigate loads at the knee.

\section{Mechanical Engineering: Orthopedic Biomechanics}

The Mechanical Engineering Department offers a course that stresses biomechanical analysis of the musculoskeletal system. In the past, a gait analysis lab had students analyze data without a 
true laboratory experience. Now, students perform trials for normal gait and for a slow jog. Students are able to experience marker placement, watch as data are collected, and learn how data are digitized. Students then perform kinetic analysis using Excel or Matlab instead of depending on Cortex to do the analysis.

The setup for the biking experiment is shown in Figure 4a, and a subject with the full marker set used during gait, jogging, and jumping is provided in Figure $4 \mathrm{~b}$.

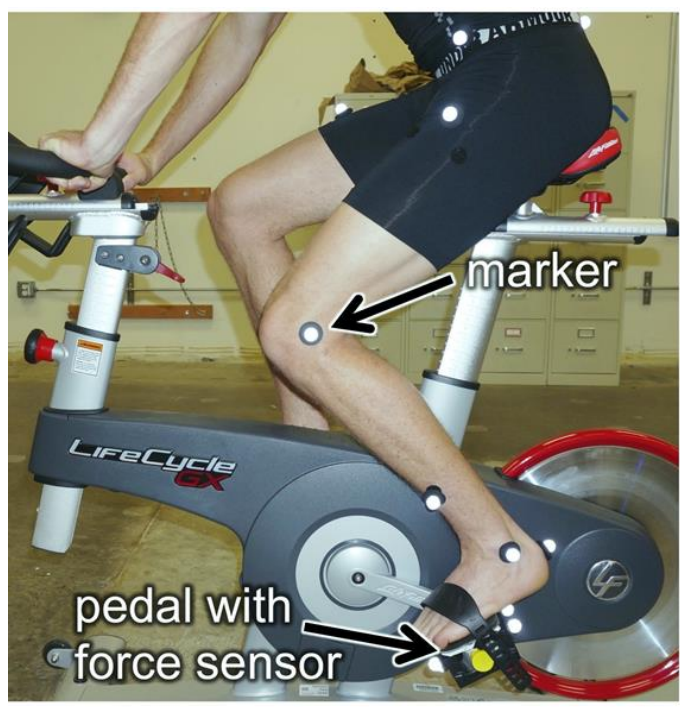

(a)
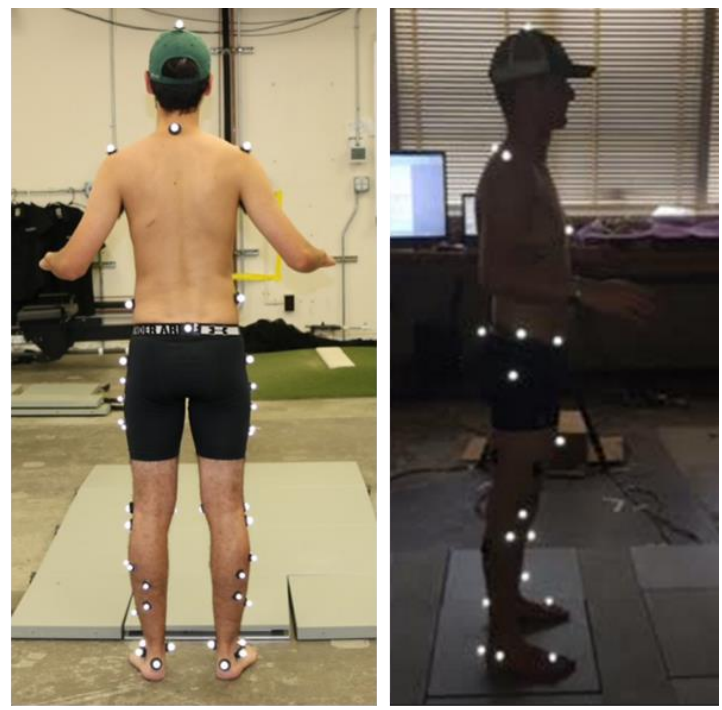

(b)

Figure 4. (a) Bicycling experimental setup (b) marker setup for gait, jogging, and jumping.

\section{Architectural Engineering}

During an Experimental Structural Analysis graduate course, students placed markers and accelerometers on structural models of buildings (see Figure 5). A small shake table provided simple sine wave inputs, as well as input data from the El Centro and Northridge earthquakes. In two of the trials a damper was placed between two of the building corners to explore their effects on building motion. Students differentiated position data from the HMBL to determine velocities and accelerations, and employed the Runge-Kutta method to find velocities and positions from the accelerometer data. 


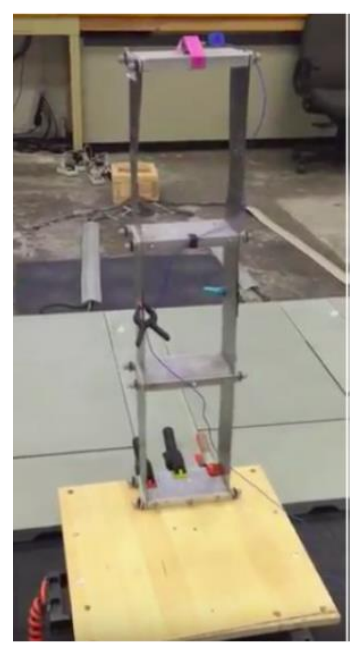

(a)

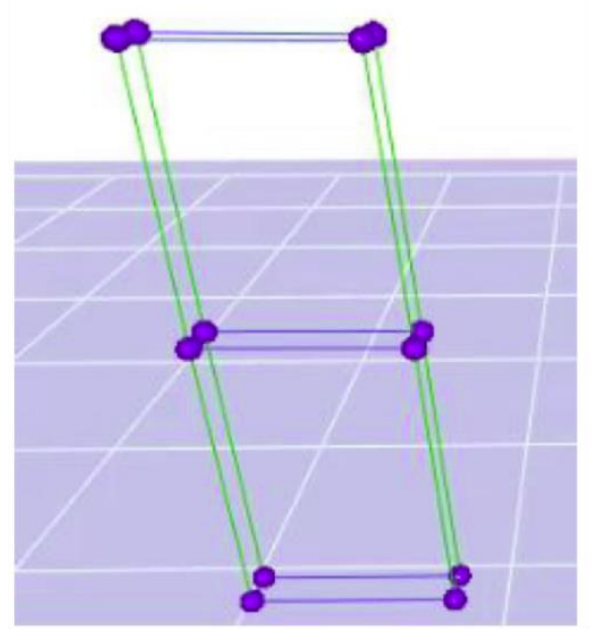

(b)

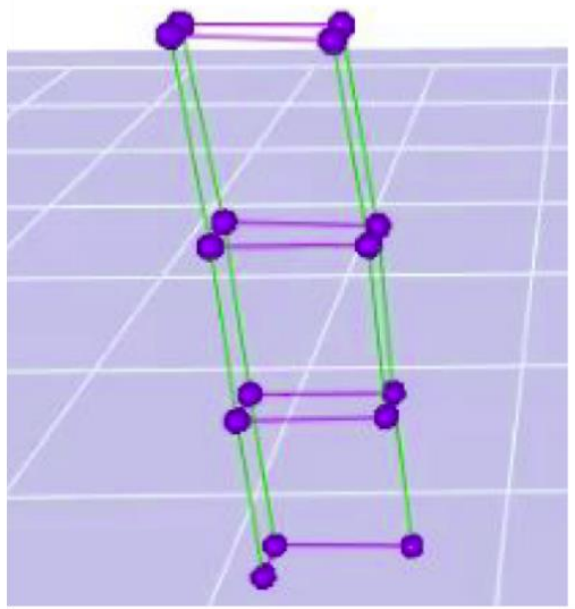

(c)

Figure 5. (a) Experimental setup with markers and accelerometers for three-story building, (b) two story building kinematic model, (c) three story kinematic building model.

\section{Methods}

For this initial analysis, we wanted to obtain feedback from the students to help us determine if we should continue using the HMBL in various courses, and to find ways to improve our use of the lab. To do this, we gave students a survey that asked agreement with the following statements:

1. Incorporating the Motion Biomechanics lab into our assignment was interesting.

2. Incorporating the Motion Biomechanics lab into our assignment helped me visualize concepts related to my course

3. Incorporating the Motion Biomechanics lab into our assignment helped me learn the material in my course.

4. Incorporating the Motion Biomechanics lab into our assignment got me interested in research.

5. Incorporating the Motion Biomechanics lab into our assignment should be incorporated into future sections of the course.

6. In future sections of the course, you should...

The first five questions used a modified Likert scale of Strongly Agree, Agree, Neither Agree nor Disagree, Disagree, or Strongly Disagree. The sixth question provided the following options:

Not include the Motion Biomechanics lab at all

Have students visit the lab for a demo, but don't include it in an assignment

Don't visit the lab, but include data collected in the lab as part of an assignment

Include the Motion Biomechanics lab in an assignment 


\section{Results}

Survey results include 41 Kinesiology Biomechanics (KINE 403) students, 86 Dynamics (ME 212) students, 18 Kinesiology Motor Learning (KINE 402) students, 49 Biomedical Engineering (BMED 410) students, 4 Intermediate Dynamics (ME326) students and 32 Orthopedic Biomechanics (ME 402) students. Student responses are shown in Figure 6.
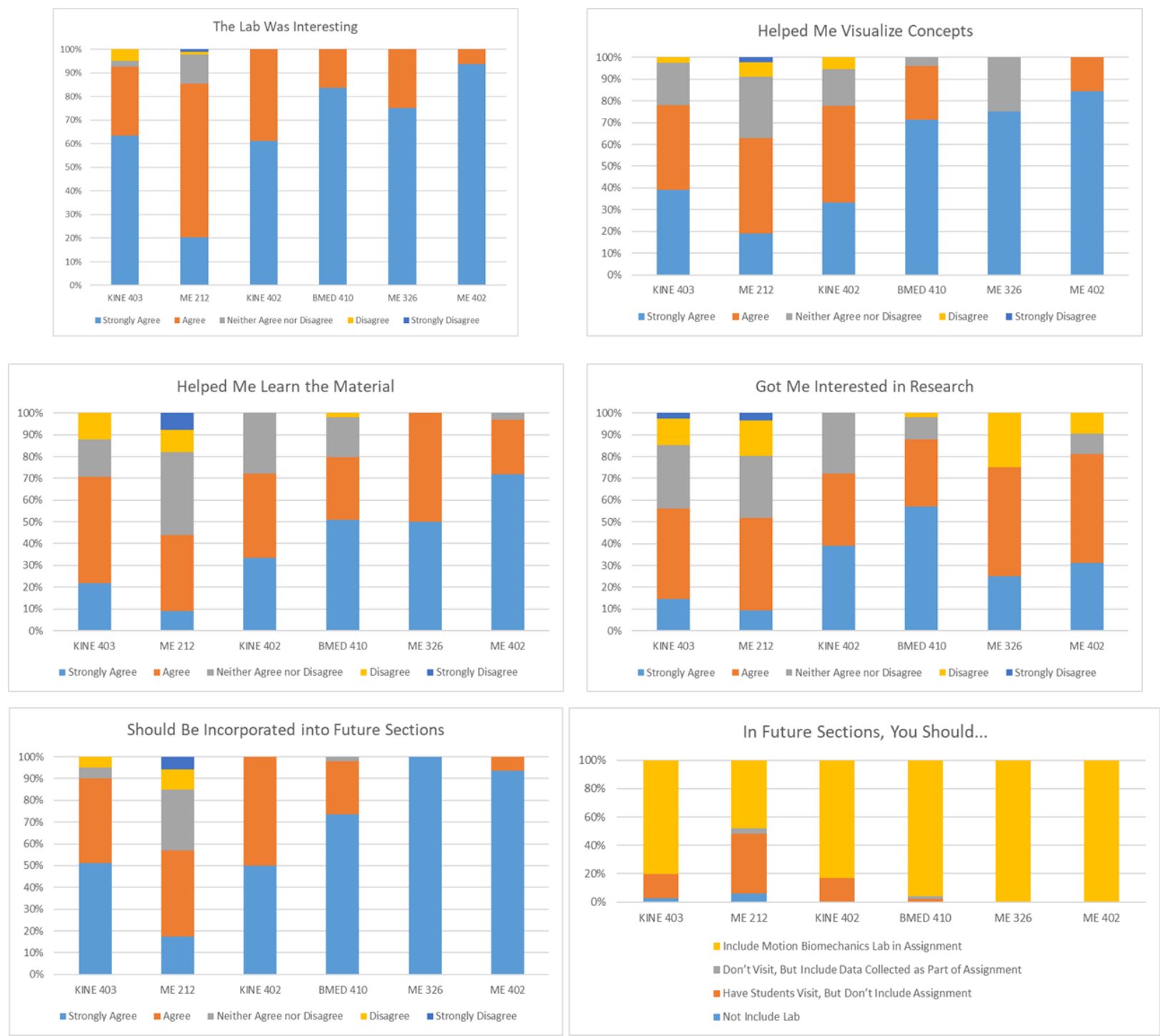

Figure 6. Student responses to survey questions. 


\section{Discussion}

In general, the response to using the HMBL was positive. The majority of students thought that the lab was interesting, and in most courses, affirmed that the lab and its data should be included in future sections of the course. The assigned projects tended to help students visualize the concepts, but perhaps not as much as we might like. Similar results were shown for the "helped me learn the material" question. A bonus result is that well over half of the participants were interested in research as a result of participating in the HMBL. We hope to see even more applicants for our biomechanics research positions as well as our Interdisciplinary Studies in Biomechanics course to be offered this spring.

Perhaps it is not surprising that the dynamics scores tended to be the lowest. The other courses all involved biomechanics content, and those students were more motivated by the lab tour and the content of the assignments. Many of the dynamics students saw this as "something extra", and didn't see the relevance to their performance on midterms or exams. Additionally, our instructions were probably a bit too open-ended for many of the students, and will need to be adjusted in the future.

Although we recognize that many institutions do not have advanced motion capture capabilities, the findings from our work can still be applied to a number of other situations. As mentioned previously, inexpensive (or free) video software (Tracker) or MatLab can be used to analyze video files. Our projects were all primarily two-dimensional, and only a handful utilized a force plate or force transducers. Our work also showed some of the benefits of working across departments. We hope that by involving students in the lab activities earlier in their education, more will be motivated to participate in research studies and to consider biomechanics and/or research careers. Finally, we hope to develop more interdisciplinary activities, such as pairing mechanical engineering and kinesiology students, to promote teamwork and interdisciplinary skills.

\section{Acknowledgements}

This work was supported by a grant from the W.M. Keck Foundation. Opinions, interpretations, conclusions, and recommendations are those of the authors.

\section{References}

[1] P. Klein, S. Gröber, J. Kuhn, A. Fleischhauer, and A. Müller, "The right frame of reference makes it simple: an example of introductory mechanics supported by video analysis of motion," European Journal of Physics, vol. 36, p. 015004, 2014.

[2] W. M. Wehrbein, "Using video analysis to investigate intermediate concepts in classical mechanics," American Journal of Physics, vol. 69, pp. 818-820, 2001.

[3] P. Laws and H. Pfister, "Using digital video analysis in introductory mechanics projects," The Physics Teacher, vol. 36, pp. 282-287, 1998. 
[4] H. Kobayashi, "Development of Motion Analysis Software for Dynamics Education," presented at the Proceedings of the ASEE Annual Conference \& Exposition, Seattle, WA, 2015.

[5] B. E. Jackson, D. J. Evangelista, D. D. Ray, and T. L. Hedrick, "3D for the people: multi-camera motion capture in the field with consumer-grade cameras and open source software," Biology open, p. bio. 018713, 2016.

[6] E. De Graaf and A. Kolmos, "Characteristics of problem-based learning," International Journal of Engineering Education, vol. 19, pp. 657-662, 2003.

[7] H. A. Diefes-Dux, T. Moore, J. Zawojewski, P. Imbrie, and D. Follman, "A framework for posing open-ended engineering problems: Model-eliciting activities," in Frontiers in Education, 2004. FIE 2004. 34th Annual, 2004, pp. F1A-3.

[8] B. Self, D. Montoya, and K. Mavrommati, "Incorporating a Motion Analysis Research Laboratory into a Dynamics Course using Model Eliciting Activities," presented at the SEFI Annual Conference, Azores, Portugal, 2017.

[9] J. Davis, S. Dick, B. P. Self, B. Wash, and J. Holton, "Observing Motor Learning and Control through Juggling and Motion Analysis: A Collaboration Between Dynamics and Kinesiology Students," in American Society for Engineering Education Pacific Southwest Section, Los Angeles, CA, 2019. 